\title{
Combined fit of spectrum and composition data as measured by the Pierre Auger Observatory
}

\author{
Armando di Matteo $^{* a}$ for the Pierre Auger Collaboration ${ }^{b}$ \\ ${ }^{a}$ INFN and Department of Physical and Chemical Sciences, University of L'Aquila, \\ L'Aquila, Italy \\ ${ }^{b}$ Observatorio Pierre Auger, Av. San Martín Norte 304, 5613 Malargüe, Argentina \\ E-mail: auger_spokespersons@fnal.gov \\ Full author list: http://www.auger.org/archive/authors_2015_06.html
}

\begin{abstract}
We present a combined fit of both flux and composition of ultra-high energy cosmic rays as measured by the Pierre Auger Observatory. The fit has been performed for energies above $5 \times 10^{18} \mathrm{eV}$, i.e. the region of the all-particle spectrum above the so-called "ankle" feature. A simple astrophysical model consisting of identical sources has been adopted, where nuclei are injected with a rigidity dependent mechanism and the sources are uniformly distributed in a comoving volume. The fit results suggest a source model characterized by relatively low maximum injection energies and hard spectral indices. The impact of different sources of systematic uncertainties in the above result is discussed.
\end{abstract}

The 34th International Cosmic Ray Conference,

30 July- 6 August, 2015

The Hague, The Netherlands

* Speaker. 


\section{Introduction and motivation}

More than half a century after their first detection, the origin of ultra-high energy cosmic rays (UHECRs), particles (mostly protons and other nuclei) reaching the Earth with energies over $10^{18} \mathrm{eV}$ up to $10^{20} \mathrm{eV}$ and beyond, is still unknown. Nevertheless, a general consensus has emerged that the most energetic cosmic rays are extragalactic, with the transition between galactic and extragalactic cosmic rays taking place somewhere between $10^{17}$ and a few times $10^{18} \mathrm{eV}$. The flux of cosmic rays above $10^{18} \mathrm{eV}$ is of the order of $1 \mathrm{~km}^{-2} \mathrm{yr}^{-1}$. Therefore, very large arrays of particle detectors are needed to study them; the largest such array is the Pierre Auger Observatory in Argentina [1]. The propagation of such particles across cosmological distances can affect their observed energy spectrum and mass composition in nontrivial ways. For this purpose several Monte Carlo codes have been developed, including CRPropa [2, 3, 4] and SimProp [5, 6, 7].

While the energy of single UHECR events can now be estimated with relatively good precision, it is impossible to determine the mass of UHECRs on an event-by-event basis. The distribution of parameters such as $X_{\max }$, the atmospheric depth at which an air shower reaches the maximum particle number, can be used to statistically estimate the mass distribution of UHECRs $[8,9]$.

In this work, we attempt to simultaneously reproduce both the Auger spectrum [10] and $X_{\max }$ [11] data with a simplified model of UHECR sources, characterized by: identical sources homogeneously distributed in a comoving volume; injection consisting only of ${ }^{1} \mathrm{H},{ }^{4} \mathrm{He},{ }^{14} \mathrm{~N}$ and ${ }^{56} \mathrm{Fe}$ nuclei, which are approximately equally spaced in $\ln A$; power-law spectrum with rigiditydependent broken exponential cutoff,

$$
\frac{\mathrm{d} N_{\mathrm{inj}, i}}{\mathrm{~d} E}= \begin{cases}J_{0} p_{i}\left(E / E_{0}\right)^{-\gamma}, & E / Z_{i}<R_{\mathrm{cut}} \\ J_{0} p_{i}\left(E / E_{0}\right)^{-\gamma} \exp \left(1-E / Z_{i} R_{\mathrm{cut}}\right), & E / Z_{i}>R_{\mathrm{cut}}\end{cases}
$$

where $J_{0}$ is a normalization factor, $E_{0}=10^{18} \mathrm{eV}, A_{i}$ and $Z_{i}$ are the mass number and atomic number of the $i$-th injected nuclide and with normalized element fractions $\sum_{i} p_{i}=1$. Such a simple model cannot reproduce measured data over their entire energy range [12]. For this reason we only fit data at energies above the ankle $\left(E \geq 10^{18.7} \mathrm{eV}\right)$ and make no hypotheses about the nature of possible extra components accounting for the rest of the sub-ankle UHECR spectrum. For examples of such hypotheses made by different authors see e.g. [13, 14, 15].

The propagated fluxes can be strongly sensitive to poorly known quantities such as the height of the far infrared peak in the extragalactic background light (EBL) spectrum and the cross sections for photodisintegration of nuclei ejecting $\alpha$ particles, as well as on approximations made in simulation codes [16]. In this work we use both SimProp and CRPropa each with several different settings to quantify the effect of such differences on

\begin{tabular}{llll} 
& MC code & $\sigma_{\text {photodisint. }}$ & EBL model \\
\hline SPG & SimProp & PSB & Gilmore 2012 \\
SPD & SimProp & PSB & Domínguez 2011 \\
STG & SimProp & TALYS & Gilmore 2012 \\
CTG & CRPropa & TALYS & Gilmore 2012 \\
CTD & CRPropa & TALYS & Domínguez 2011 \\
CGD & CRPropa & Geant4 & Domínguez 2011
\end{tabular}

Table 1: The various propagation models we used (see Ref. [16] and references therein for details) our fit (see Table 1). Other EBL models such as Kneiske 2004 [17] or Stecker 2005 [18, 19] give similar results. 


\section{Fit results and physical parameters}

The data we attempt to fit consist of 15 measurements of the UHECR energy spectrum in $\log _{10}(E / \mathrm{eV})$ bins of width 0.1 from 18.7 to 20.2 [10], and 110 non-zero measurements of the $X_{\max }$ distribution in $\log _{10}(E / \mathrm{eV})$ bins of width 0.1 from 18.7 to 19.5 plus one of width 0.5 from 19.5 to 20.0 and $X_{\max }$ bins of width $20 \mathrm{~g} / \mathrm{cm}^{2}$ [11]. We approximate the probability distribution of each point of the spectrum as a Gaussian with the standard deviation corresponding to the measurement statistical uncertainty, whereas for the $X_{\max }$ distributions we use a multinomial distribution over the $X_{\max }$ bins for each energy bin, where the mass distributions at the Earth from the simulation outputs are converted to the $X_{\max }$ distributions via a Gumbel parameterization [20] based on the EPOS-LHC [21] model of UHECR-air interactions.

The free parameters of the fit are: the injection normalization factor $J_{0}$, the injection spectral index $\gamma$, the cutoff rigidity $R_{\text {cut }}$, and the element fractions at injection (three free parameters $p_{\mathrm{H}}$, $p_{\mathrm{He}}, p_{\mathrm{N}}$; the fourth is bound by $\left.p_{\mathrm{Fe}}=1-p_{\mathrm{H}}-p_{\mathrm{He}}-p_{\mathrm{N}}\right)$. In total, we have 125 non-zero data points and 6 free parameters.

Using the propagation model listed as SPG in Table 1, we find the best fit at $\gamma=0.94_{-0.10}^{+0.09}$, $R_{\text {cut }}=10^{18.67 \pm 0.03} \mathrm{~V}$, with a deviance ${ }^{1}$ (generalized $\chi^{2}$ ) per degree of freedom $D_{\min } / n=178.5 / 119 .^{2}$ To assess the statistical significance of this, we repeated the fit using $10^{4}$ mock data sets for the spectrum and $X_{\max }$ distributions generated according to the best-fit model and the same statistic as the real data, and found that $D_{\min }$ exceeds 178.5 for $p=2.6 \%$ of the mock data sets, indicating a slightly statistically significant deviation of the data from the model. The normalization $J_{0}$ we find corresponds to a total emissivity $\mathscr{L}_{0}=5.15 \times 10^{44} \mathrm{erg} / \mathrm{Mpc}^{3} / \mathrm{yr}$, and those for the single elements $\mathscr{L}_{\mathrm{He}}=0.289 \mathscr{L}_{0}, \mathscr{L}_{\mathrm{N}}=0.656 \mathscr{L}_{0}$, and $\mathscr{L}_{\mathrm{Fe}}=0.055 \mathscr{L}_{0}$.

In Fig. 1 we show the deviance of our fit as a function of $\left(\gamma, R_{\text {cut }}\right)$, where for each point of the profile likelihood plane the values of the remaining paramenters $\left(J_{0}\right.$ and $\left.p_{i}\right)$ are chosen so as to minimize the deviance (marginalized). The best fit can be seen to be part of a long 'valley' extending to lower values of $\gamma$ and $R_{\text {cut }}$ approximately along the shown curve. There also is a second local minimum at $\gamma \approx 2$, but it is much worse than the global minimun $\left(D_{2}-D_{1}=56.5\right.$, corresponding to a $7.5 \sigma$ exclusion; $p=5 \times 10^{-4}$ from mock data sets), mainly due to predicted $X_{\max }$ distributions at most energies that are broader than observed.

The corresponding simulated spectra and the mean and variance of the simulated $X_{\max }$ distributions are shown in the left panels of Fig. 2 for the best fit and in the right panels for the local minimum at $\gamma \approx 2$.

\section{Systematic uncertainty due to the propagation}

Certain assumptions about the processes affecting the propagation of UHECRs can result in substantially different spectra at the Earth for a given spectrum at injection [16]. To study the effect

\footnotetext{
${ }^{1}$ The deviance is defined as $D=-2 \ln \left(L / L_{\mathrm{sat}}\right)$, where $L$ is the likelihood of the model considered and $L_{\mathrm{sat}}$ is that of a hypothetical model perfectly reproducing the data. It coincides with the usual $\chi^{2}$ statistic for Gaussian likelihoods.

${ }^{2}$ The uncertainties given for $\gamma$ and $R_{\text {cut }}$ correspond to the intervals where $D \leq D_{\min }+1$ (68\% confidence). For the fit with propagation model SPG, the correctness of errors has been checked with a posteriori errors obtained from mock data sets, which were also used for the uncertainties on $p_{i}$ given in Fig. 1.
} 


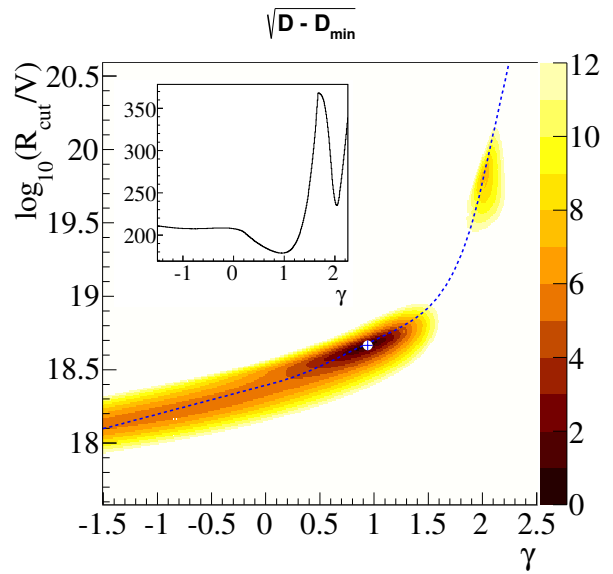

\begin{tabular}{lrr} 
model SPG & best fit & 2nd local min \\
\hline$J_{0}\left[\mathrm{eV}^{-1} \mathrm{Mpc}^{-3} \mathrm{yr}^{-1}\right]$ & $7.17 \times 10^{18}$ & $4.53 \times 10^{19}$ \\
$\gamma$ & $0.94_{-0.10}^{+0.09}$ & 2.03 \\
$\log _{10}\left(R_{\text {cut }} / \mathrm{V}\right)$ & $18.67 \pm 0.03$ & 19.84 \\
$p_{\mathrm{H}}$ & $0.0^{+29.9 \%}$ & $0.0 \%$ \\
$p_{\mathrm{He}}$ & $62.0_{-22.2}^{+3.5} \%$ & $0.0 \%$ \\
$p_{\mathrm{N}}$ & $37.2_{-12.6}^{+4.2} \%$ & $94.2 \%$ \\
$p_{\mathrm{Fe}}$ & $0.8_{-0.3}^{+0.2} \%$ & $5.8 \%$ \\
\hline$D / n$ & $178.5 / 119$ & $235.0 / 119$ \\
$D(J), D\left(X_{\max }\right)$ & $18.8,159.8$ & $14.5,220.5$ \\
$p$ & $2.6 \%$ & $5 \times 10^{-4}$
\end{tabular}

Figure 1: Left: $\sqrt{D-D_{\min }}$ where $D$ is the profile deviance as a function of $\left(\gamma, R_{\text {cut }}\right)$ and $D_{\min }$ is the best-fit deviance. Each coloured area corresponds to $1 \sigma, 2 \sigma, \ldots$ confidence intervals. The inset shows the values of $D$ along the dotted curve. Right: best-fit and second local minimum parameters for model SPG.
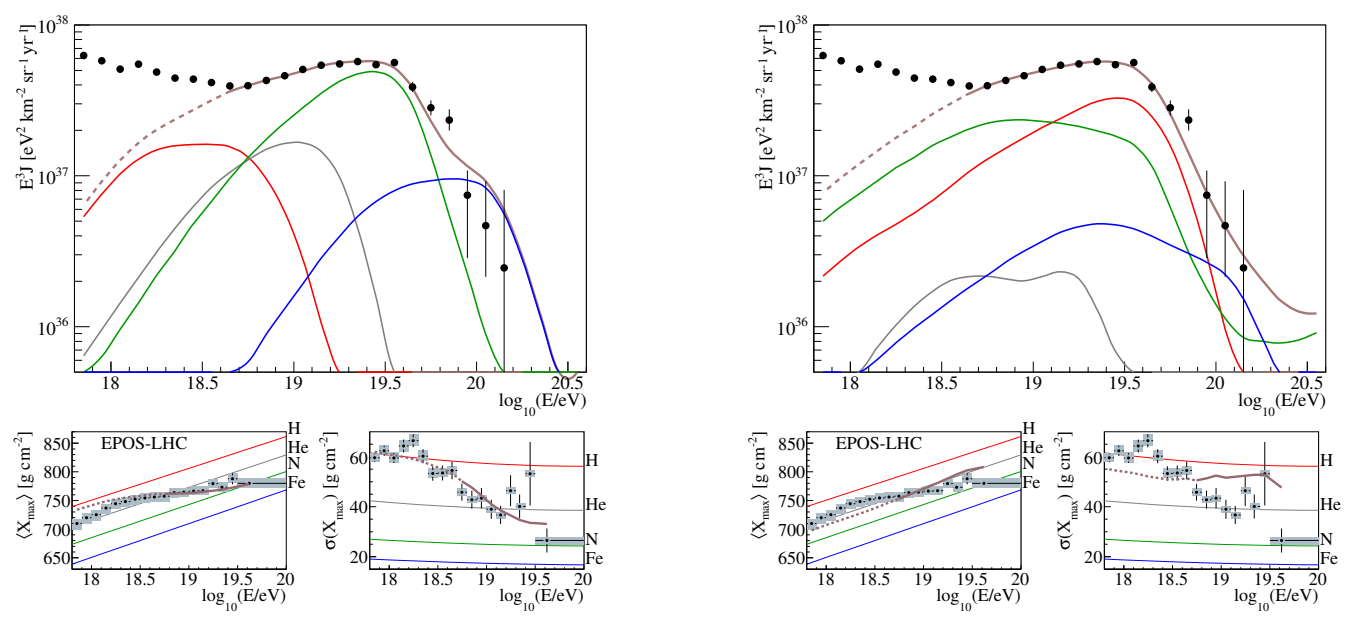

Figure 2: Top: simulated energy spectrum of UHECRs (multiplied by $E^{3}$ ) at the top of the Earth's atmosphere with the best-fit parameters (left) and the local minimum at $\gamma \approx 2$ (right) for model SPG, along with Auger data points [10]. Partial spectra are grouped according to the mass number as follows: $A=1$ (red), $2 \leq A \leq 4$ (grey), $5 \leq A \leq 26$ (green), $27 \leq A$ (blue), total (brown). Bottom: average and standard deviation of the $X_{\max }$ distribution as predicted (assuming EPOS-LHC UHECR-air interactions) for the model predictions in the two scenarios (brown), pure ${ }^{1} \mathrm{H}$ (red), ${ }^{4} \mathrm{He}$ (grey), ${ }^{14} \mathrm{~N}$ (green) and ${ }^{56} \mathrm{Fe}$ (blue). Only the energy range where the brown lines are solid is included in the fit.

of this on our results, we repeated the fit described in the previous section for each of the various propagation models listed in Table 1. The results are shown in Table 2.

From Fig. 3, it can be seen that the relationship between $\gamma$ and $R_{\text {cut }}$ and the position of the second local minimum are very similar from one model to another, but the position of the best fit within the 'valley' and the height of the 'ridge' between the two local minima are strongly modeldependent. Furthermore, propagation models with lower photodisintegration rates ${ }^{3}$ tend to result in better fits to the Auger data, except at very low values of $\gamma$ and $R_{\text {cut }}$.

\footnotetext{
${ }^{3}$ The Domínguez EBL model has a stronger far infrared peak than the Gilmore model, and TALYS predicts sizeable
} 


\begin{tabular}{l|lll} 
& $\gamma(1 \mathrm{st} \min )$ & $\log _{10}\left(R_{\mathrm{cut}} / \mathrm{V}\right)$ & $D_{D\left(X_{\max }\right)}^{D(J)}$ \\
\hline SPG & $+0.94_{-0.10}^{+0.09}$ & $18.67 \pm 0.03$ & $178.5_{159.8}^{18.8}$ \\
SPD & $-0.45^{2} \pm 0.41$ & $18.27_{-0.06}^{+0.07}$ & $193.4_{172.3}^{21.1}$ \\
STG & $+0.69_{-0.06}^{+0.07}$ & $18.60 \pm 0.01$ & $176.9_{157.5}^{19.5}$ \\
CTG & $+0.73_{-0.07}^{+0.07}$ & $18.58 \pm 0.01$ & $195.3_{161.6}^{33.6}$ \\
CTD & $-1.06_{-0.22}^{+0.29}$ & $18.19_{-0.02}^{+0.04}$ & $192.3_{171.2}^{21.2}$ \\
CGD & $-1.29_{*}^{+0.38}$ & $18.18_{-0.04}^{+0.06}$ & $192.5_{173.3}^{19.2}$ \\
\multicolumn{3}{c}{ *This interval extends all the way down to -1.5,} \\
\multicolumn{3}{c}{ the lowest value of $\gamma$ we considered. }
\end{tabular}

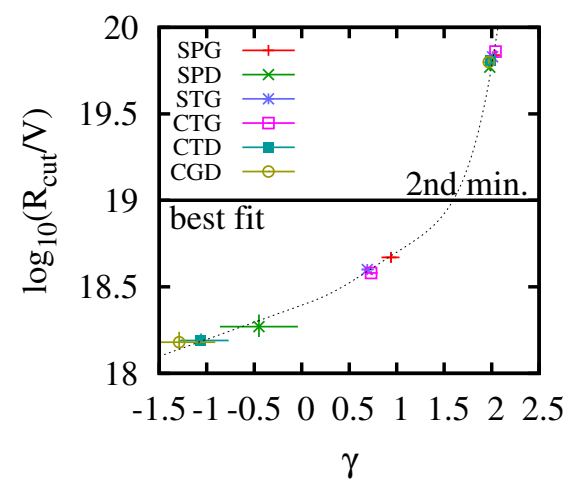

Table 2: Best-fit parameters and 68\% uncertainties for the various propagation models we used (see Table 1). In the right panel local minima at $\gamma \approx 2$ are also shown. The dotted line is the same as in Fig. 1.
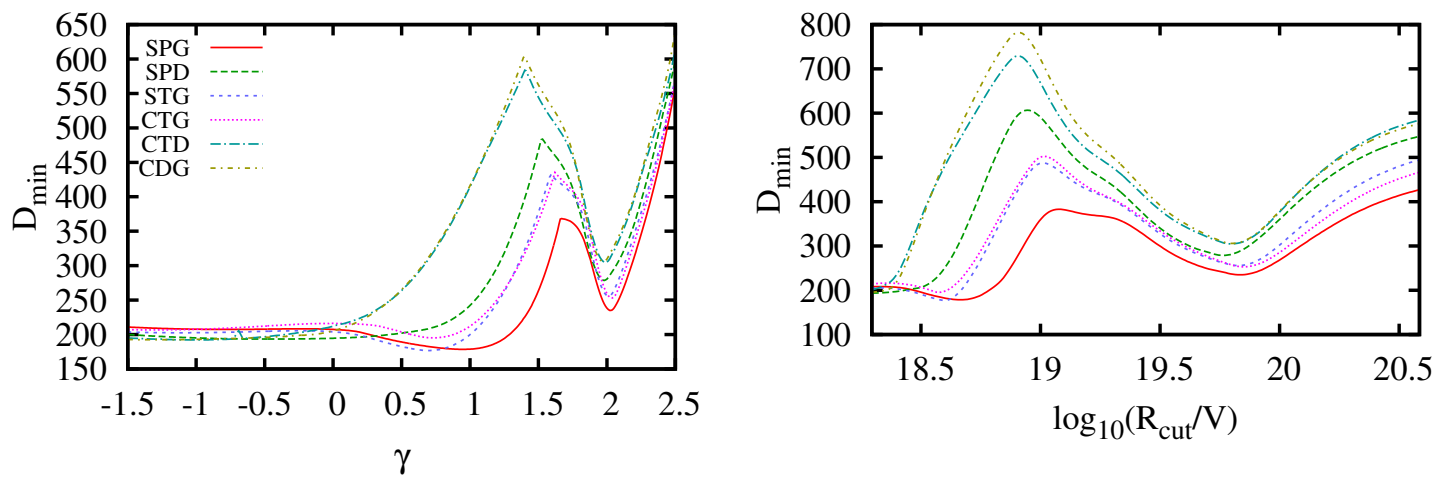

Figure 3: $D$ for each value of $\gamma$ (first panel) and $R_{\text {cut }}$ (second panel) for each propagation model, where the other parameters are chosen so as to minimize $D$

\section{Effect of systematic uncertainties of the measurements}

To study the effect of the systematic uncertainty of the energy scale of the Pierre Auger Observatory, we repeated the fit using the propagation model A shifting the energy of all measured data points by $\pm 14 \%$, corresponding the systematic uncertainty of the energy scale, and/or shifting the $X_{\max }$ scale by the systematical uncertainties. ${ }^{4}$. The resulting best fit parameters are shown in Table 3 and the relationships between the parameters and the deviance are shown in Fig. 4.

Except at very high values of $R_{\text {cut }}$, the fit is improved by shifting the energies downwards and worsened by shifting them upwards. This effect is largely due to the data points in the measured spectrum around $10^{19.8} \mathrm{eV}$, being shifted towards or away from the descent in the simulated propagated nitrogen spectrum due to photodisintegration interactions. As for the $X_{\max }$ scale, lowering it in higher values of $\gamma$ and $R_{\text {cut }}$ and vice versa. Furthermore, lowering the $X_{\max }$ scale improves the fit and raising it worsens it.

cross sections for certain photodisintegration channels (largely in excess of the available measurements) which are neglected altogether in PSB.

${ }^{4}$ The systematical uncertainties on $X_{\max }$ are asymmetric and slightly dependent on energy, ranging from 6.9 to $9.4 \mathrm{~g} / \mathrm{cm}^{2}$. See Table IV in Ref. [11] for details. 


\begin{tabular}{|c|c|c|c|}
\hline $\begin{array}{c}\gamma \\
\log _{10}\left(R_{\text {cut }} / \mathrm{V}\right) \\
D(J) \\
D\left(X_{\max }\right)\end{array}$ & $-14 \%$ & $\begin{array}{c}\text { nominal } \\
\text { energy } \\
\text { scale }\end{array}$ & $+14 \%$ \\
\hline$-1 \sigma_{\text {syst }}$ & $\begin{array}{c}+1.32_{-0.07}^{+0.05} \\
18.68_{-0.04}^{+0.05} \\
157.4_{148.4}^{9.0} \\
\end{array}$ & $\begin{array}{c}+1.35 \pm 0.05 \\
18.73 \pm 0.02 \\
172.1_{153.7}^{18.4}\end{array}$ & $\begin{array}{c}+1.39_{-0.04}^{+0.05} \\
18.78 \pm 0.01 \\
203.5_{157.0}^{46.5}\end{array}$ \\
\hline $\begin{array}{c}\text { nominal } \\
X_{\max } \\
\text { scale }\end{array}$ & $\begin{array}{c}+0.90_{-0.15}^{+0.10} \\
18.64_{-0.04}^{+0.03} \\
165.5_{156.1}^{9.3}\end{array}$ & $\begin{array}{c}+0.94_{-0.10}^{+0.09} \\
18.67 \pm 0.03 \\
178.5_{159.8}^{18.8}\end{array}$ & $\begin{array}{c}+0.98_{-0.11}^{+0.10} \\
18.70 \pm 0.03 \\
214.9_{164.5}^{50.4}\end{array}$ \\
\hline$+1 \sigma_{\text {syst }}$ & $\begin{array}{l}\leq-1.50 \\
\leq 18.22 \\
\leq 207.0\end{array}$ & $\begin{array}{l}\leq-1.50 \\
\leq 18.24 \\
\leq 217.2\end{array}$ & $\begin{array}{c}-1.34_{*}^{+0.31} \\
18.28_{-0.08}^{+0.05} \\
256.0_{200.4}^{55.7}\end{array}$ \\
\hline
\end{tabular}

*This interval extends all the way down to -1.5 , the lowest value of $\gamma$ we considered.

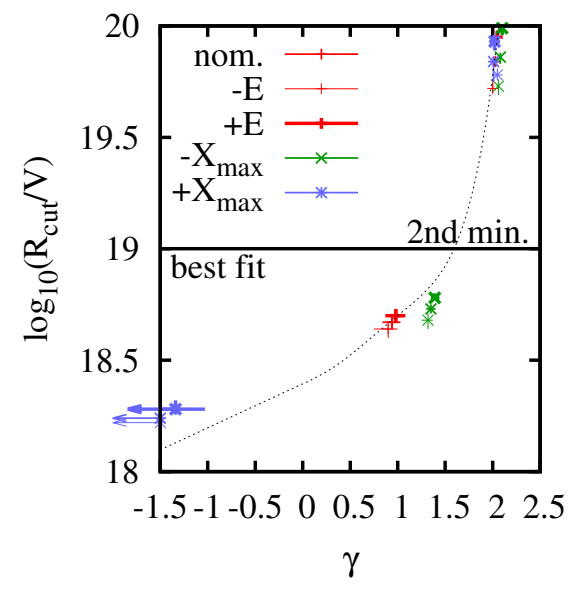

Table 3: Same as in Table 2, using propagation model SPG and shifting the Auger energy and/or $X_{\max }$ scales
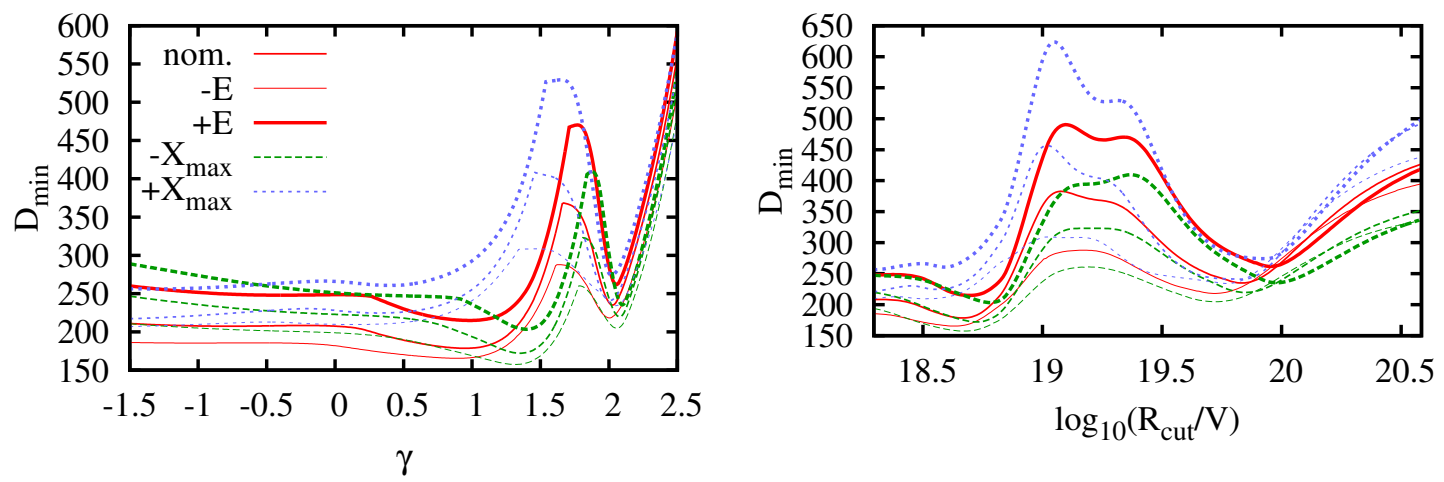

Figure 4: Same as in Fig. 3, using propagation model SPG and shifting the Auger energy and/or $X_{\max }$ scales

\section{Effect of UHECR-air interaction models}

In order to study the effect of different models of UHECR-air interactions, we repeated our fit with propagation model SPG using Sibyll 2.1 [22] and QGSJet II-04 [23] instead of EPOS-LHC. The results are shown in Table 4 and in Fig. 5.

The use of Sibyll 2.1, and to an even larger extent QGSJet II-04, worsens the fit at all considered values of $\gamma$ and $R_{\text {cut }}$; in addition, the low- $\gamma$ minimum is pushed down to

\begin{tabular}{l|lll} 
& $\gamma(1$ st min $)$ & $\log _{10}\left(R_{\text {cut }} / \mathrm{V}\right)$ & $D_{D\left(X_{\max }\right)}^{D(J)}$ \\
\hline $\mathrm{E}$ & $+0.94_{-0.10}^{+0.09}$ & $18.67 \pm 0.03$ & $178.5_{159.8}^{18.8}$ \\
$\mathrm{~S}$ & $\leq-1.50$ & $\leq 18.27$ & $\leq 256.8$ \\
$\mathrm{Q}$ & $\leq-1.50$ & $\leq 18.28$ & $\leq 344.3^{*}$
\end{tabular}

*At least in the $\gamma$ range we considered, this minimum is actually worse than that at $\gamma \approx 2$ for this model.

Table 4: Same as Table 2, using propagation model SPG and various UHECR-air interaction models (E: EPOS-LHC, S: Sibyll 2.1, Q: QGSJet II-04) values of $\gamma$ outside the range we considered, and with QGSJet II-04 the high- $\gamma$ local minimum is better than at least the visible portion of the low- $\gamma$ one. 

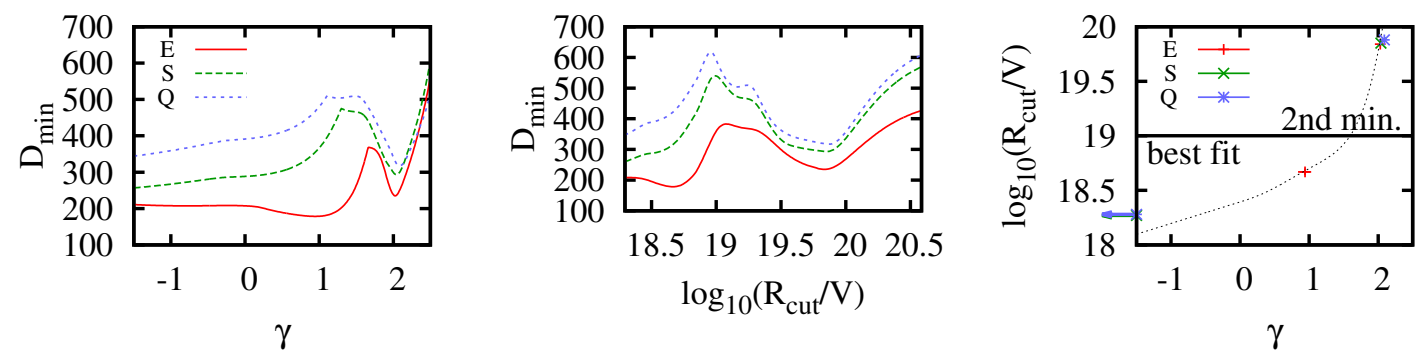

Figure 5: Same as in Fig. 3, using propagation model SPG and various UHECR-air interaction models (E: EPOS-LHC, S: Sibyll 2.1, Q: QGSJet II-04)

\section{Conclusions}

When interpreted with a simple model of UHECR injection, the Auger data are best fitted by very hard $(\gamma \lesssim 1)$ injection spectra and the flux is mostly limited by the maximum energy at the sources. The local minimum with $\gamma \approx 2$ and large maximum rigidity, which is more in line with standard models of cosmic ray acceleration, predicts wider distributions of UHECR masses at each energy than observed in the data. This conclusion is robust with respect to all the model variations we considered, but the position of the best fit is strongly sensitive to the details of the propagation, though in all cases it is in the same curved strip of the $\left(\gamma, R_{\text {cut }}\right)$ plane. The uncertainty due to our ignorance of details of the propagation are much larger than that due to the statistical uncertainty of measured data.

As for the goodness of fit, it is better for models of UHECR propagation with lower photodisintegration rates (Gilmore 2012 EBL model, PSB cross sections) than with higher rates (Domínguez 2011 EBL model, TALYS cross sections), better for models of UHECR-air interactions predicting deeper showers (EPOS-LHC) than shallower ones (QGSJet II-04), and better for the cases when the Auger energy and $X_{\max }$ scale are assumed to be lower than the nominal ones.

Other possible ways of improving the agreement with measured data, such as considering more injection masses, considering non-uniform source distributions, or more complicated injection spectra, are outside the scope of this work.

\section{References}

[1] Pierre Auger Collaboration, The Pierre Auger Cosmic Ray Observatory, accepted for publication in Nucl. Instrum. Meth. A (2015) [arXiv:1502.01323].

[2] E. Armengaud et al., CRPropa: a numerical tool for the propagation of UHE cosmic rays, gamma-rays and neutrinos, Astropart. Phys. 28 (2007) 463-471, [astro-ph / 0603675 ].

[3] K.-H. Kampert et al., CRPropa 2.0 - a Public Framework for Propagating High Energy Nuclei, Secondary Gamma Rays and Neutrinos, Astropart. Phys. 42 (2013) 41-51, [arXiv: 1206.3132 ].

[4] R. A. Batista et al., CRPropa 3.0 - a Public Framework for Propagating UHE Cosmic Rays through Galactic and Extragalactic Space, Proc. 33rd ICRC (2013) [arXiv: 1307 . 2643].

[5] R. Aloisio et al., SimProp: a Simulation Code for Ultra High Energy Cosmic Ray Propagation, JCAP 1210 (2012) 007, [arXiv:1204.2970]. 
[6] R. Aloisio et al., Propagation of UHECRs in cosmological backgrounds: some results from SimProp, Proc. 33rd ICRC (2013) [arXiv: 1307.3895].

[7] R. Aloisio et al., SimProp v2r2: a Monte Carlo simulation to compute cosmogenic neutrino fluxes, Gran Sasso Science Institute internal note GSSI/PHYS/2015.0042 (2015) [arXiv: 1505.01347 ].

[8] Pierre Auger Collaboration, Interpretation of the Depths of Maximum of Extensive Air Showers Measured by the Pierre Auger Observatory, JCAP 1302 (2013) 026, [arXiv: 1301.6637 ].

[9] Pierre Auger Collaboration, Depth of maximum of air-shower profiles at the Pierre Auger Observatory. II. Composition implications, Phys. Rev. D90 (2014) 122006, [arXiv: 1409.5083 ].

[10] Pierre Auger Collaboration, The Pierre Auger Observatory: Contributions to the 33rd International Cosmic Ray Conference (ICRC 2013), Proc. 33rd ICRC (2013) [arXiv:1307.5059].

[11] Pierre Auger Collaboration, Depth of maximum of air-shower profiles at the Pierre Auger Observatory. I. Measurements at energies above $10^{17.8}$ eV, Phys. Rev. D90 (2014) 122005, [arXiv:1409.4809].

[12] Pierre Auger Collaboration, Combined fit of spectrum and composition data as measured by the Pierre Auger Observatory, in preparation.

[13] R. Aloisio, V. Berezinsky, and P. Blasi, Ultra high energy cosmic rays: implications of Auger data for source spectra and chemical composition, JCAP 1410 (2014) 020, [arXiv : 1312 . 7459].

[14] N. Globus, D. Allard, and E. Parizot, A complete model of the CR spectrum and composition across the Galactic to Extragalactic transition, submitted to Phys. Rev. D Rapid Communications (2015) [arXiv:1505.01377].

[15] M. Unger, G. R. Farrar, and L. A. Anchordoqui, Origin of the ankle in the ultra-high energy cosmic ray spectrum and of the extragalactic protons below it, arXiv preprints (2015) [arXiv:1505.02153].

[16] R. Alves Batista et al., Effects of uncertainties in simulations of extragalactic UHECR propagation, using CRPropa and SimProp, submitted to JCAP (2015) [arXiv:1508.01824].

[17] T. M. Kneiske et al., Implications of cosmological gamma-ray absorption. 2. Modification of gamma-ray spectra, Astron. Astrophys. 413 (2004) 807-815, [astro-ph/ 0309141 ].

[18] F. W. Stecker, M. Malkan, and S. Scully, Intergalactic photon spectra from the far IR to the UV Lyman limit for $0<z<6$ and the optical depth of the universe to high energy gamma-rays, Astrophys. J. 648 (2006) 774-783, [astro-ph/ 051044 9].

[19] F. W. Stecker, M. Malkan, and S. Scully, Corrected Table for the Parametric Coefficients for the Optical Depth of the Universe to Gamma-rays at Various Redshifts, Astrophys. J. 658 (2007) 1392, [astro-ph/0612048].

[20] M. De Domenico et al., Reinterpreting the development of extensive air showers initiated by nuclei and photons, JCAP 1307 (2013) 050, [arXiv: 1305.2331 ].

[21] K. Werner, F.-M. Liu, and T. Pierog, Parton ladder splitting and the rapidity dependence of transverse momentum spectra in deuteron-gold collisions at RHIC, Phys. Rev. C74 (2006) 044902, [hep-ph/0506232].

[22] E.-J. Ahn et al., Cosmic ray interaction event generator SIBYLL 2.1, Phys. Rev. D80 (2009) 094003, [arXiv:0906.4113].

[23] S. Ostapchenko, Non-linear screening effects in high energy hadronic interactions, Phys. Rev. D74 (2006) 014026, [hep-ph/ 0505259 ]. 